\title{
Modeling of the 2D self-assembly of tripod-shaped functional molecules with patchy interaction centers
}

\author{
Wojciech Rżysko ${ }^{1}$ Damian Nieckarz ${ }^{2} \cdot$ Paweł Szabelski $^{2}$
}

Received: 30 October 2018 / Revised: 30 November 2018 / Accepted: 5 December 2018 / Published online: 11 December 2018

(C) The Author(s) 2018

\begin{abstract}
Surface-confined self-assembly of star-shaped functional molecules has been recently recognized as a promising method to fabricate extended superstructures with predefined architectures and physico-chemical functions. In this work we use the Monte Carlo (MC) method to explore 2D self-assembly of rigid tripod-shaped tectons equipped with patchy interaction centers located at the ends of molecular arms. These angular interaction zones represent terminal functional groups, which due to some flexibility/extended size, can provide intermolecular bonds which does not have to be collinear with the arms. Our main focus is on the effect of angular diameter of the interaction centers on the morphology of the simulated assemblies. Moreover, we explore the influence of molecular backbone symmetry on the periodicity and connectivity of the resulting adsorbed superstructures. The obtained results are compared with their selected counterparts from lattice MC simulations. It is demonstrated that a suitable choice of the size of the interaction centers and backbone aspect ratio allows for directing the self-assembly towards 2D networks comprising pores of different size and shape. The results from our theoretical modeling can be helpful in designing new functional organic building blocks able to form supramolecular networks with desired structural properties.
\end{abstract}

Keywords Self-assembly $\cdot$ Adsorbed overlayers $\cdot$ Functional molecules $\cdot$ Monte Carlo simulation $\cdot$ 2D porous networks

\section{Introduction}

Recent progress in methods of creation and imaging of adsorbed systems has enabled directing the self-assembly of molecular building blocks into low-dimensional superstructures with tunable geometric and physico-chemical properties. A central question related to the controlled fabrication of ordered molecular assemblies on solid surfaces is how individual features of a building block, including its size, shape and interaction pattern, affect the resulting superstructure. To answer this question, test experiments are usually required in which different probe molecular units are synthesized, and their self-assembly is monitored with such

Paweł Szabelski

szabla@vega.umcs.lublin.pl

1 Department for the Modeling of Physico-Chemical Processes, Maria-Curie Skłodowska University, Pl. M.C. Skłodowskiej 3, 20-031 Lublin, Poland

2 Department of Theoretical Chemistry, Maria-Curie Skłodowska University, Pl. M.C. Skłodowskiej 3, 20-031 Lublin, Poland imaging techniques as the scanning tunneling microscopy (STM) (De Feyter and De Schryver 2005). To date, numerous examples of ordered adsorbed structures ranging from simple densely packed motifs (Trawny et al. 2015), nanoporous networks (Kudernac et al. 2009; Tahara et al. 2011; Bischoff et al. 2016) to openwork fractal aggregates (Shang et al. 2015; Zhang et al. 2015) have been realized using functional organic building blocks. In these instances, often linear (Kühne et al. 2009a, b), bent-rod (Fan et al. 2014, 2016; Shang et al. 2015; Zhang et al. 2015) and star shaped (Kudernac et al. 2009; Dienstmaier et al. 2010; Shi et al. 2011; Tahara et al. 2011; Ciesielski et al. 2013; Bischoff et al. 2016) polyphenyl molecules with suitably attached functional groups, including nitrile (Kühne et al. 2009a, b; Shang et al. 2015), pyridyl (Shi et al. 2011; Ciesielski et al. 2013), carboxylic (Dienstmaier et al. 2010), aldehyde (Zhang et al. 2015) and others (Shang et al. 2015) have been used. The aforementioned superstructures have been often sustained by intermolecular hydrogen bonds (Kudernac et al. 2009; Kühne et al. 2009a, b; Dienstmaier et al. 2010; Ciesielski et al. 2013) but they can be also stabilized by metal-ligand coordination occurring in mixed metal-organic 
systems (Shi et al. 2011; Fan et al. 2014, 2016), halogen bonds (Shang et al. 2015) or even by van der Waals interactions between the star-shaped molecules equipped with alkyl chain arms (Tahara et al. 2006). An important observation from the structure formation in adsorbed overlayers comprising functional organic molecules is that chemically different building blocks are often able to create isostructural 2D patterns. This refers especially to the nanoporous networks, for example to those with hexagonal void spaces, which can be formed by such different tripod-shaped molecules like tricarboxylic acids (Dienstmaier et al. 2010), tectons equipped with propyloxy (Bertrand et al. 2011) and amine groups (Liu et al. 2013). In this case, similar molecular shape and intramolecular distribution of interaction centers within the building block are the deciding factors which direct the self-assembly towards superstructures of the same type. This finding allows for the identification and classification of the main qualitative features which are necessary for a molecule to form adsorbed assemblies with predefined morphology. Such information can largely facilitate synthesis of the corresponding molecular tectons and for that reason, formulation of general structure-property relations has been important from the practical point of view. Obtaining relation of this kind, apart from experimental tests (Kaposi et al. 2016), can be also based on theoretical methods in which intrinsic molecular parameters can be easily adjusted and diverse molecular pattern can be systematically designed.

A particularly effective theoretical approach to study the general structure-property relation in the 2D self-assembled systems are the coarse-grained simulation methods in which molecular tectons are mapped in a simplified way, for example as collections of interconnected discrete units (segments). In this case, a model building block can represent a wide class of chemically different molecular tectons which are similar in size/shape and location of interaction centers. The coarsegrained picture mentioned above has been frequently used in the Monte Carlo (MC) simulations of adsorbed systems on solid substrates. This refers to simple rod-like molecules (Matoz-Fernandez et al. 2008; Centres and Ramirez-Pastor 2009; Nieckarz and Szabelski 2013) as well as to molecules with more complex structures, including cross-shaped units resembling porphyrins and phthalocyanines ( $\mathrm{Li}$ and $\mathrm{Lin} 2011$; Akimenko et al. 2015; Kasperski et al. 2015; Bischoff et al. 2016; Gorbunov et al. 2017), tripod-shaped tectons corresponding to tricarboxylic acid derivatives (Ibenskas and Tornau 2012; Misiunas and Tornau 2012; Šimenas et al. 2015), dehydrobenzoannulenes (DBAs) (Szabelski et al. 2010; Adisoejoso et al. 2012) and molecules bearing pyridyl (Ciesielski et al. 2013) and nitrile (Copie et al. 2015) groups. The results from these theoretical studies demonstrate that the MC calculations, performed even for the simplified molecular representations, are able to reproduce correctly formation and coexistence of various adsorbed phases observed experimentally, especially the planar nanoporous networks observed either in ultra high vacuum or at the liquid/solid interface. Moreover, with the help of the coarse-grained MC simulations it was possible to predict new supramolacular structures, for example, such exotic as the fractal metal-organic aggregates (Nieckarz and Szabelski 2014) whose existence was latter exactly confirmed experimentally (Sun et al. 2015; Li et al. 2015).

As it has been often observed in the experiment, the interactions cementing 2D molecular superstructures are usually short-ranged and highly directional (e.g. hydrogen bonding, metal-ligand coordination) (Kudernac et al. 2009; Kühne et al. 2009a, b; Dienstmaier et al. 2010; Shi et al. 2011; Ciesielski et al. 2013; Fan et al. 2014, 2016). This property facilitates modeling of these superstructures, as for the contributing molecules a set of discrete interaction directions can be usually assigned in order to map the interaction pattern with reasonable accuracy. Moreover, for those building blocks whose symmetry matches symmetry of the adsorbing surface, for example tripod-shaped molecules on graphite or metallic (111) substrates, the discrete interaction directions can be assigned according to the symmetry imposed by the (triangular) lattice representing the real surface. This situation refers to these adsorbed assemblies for which the corrugation of the surface-molecule potential results in a limited number of on-surface configurations of a single molecule and thus in the limited number of bimolecular connections differing in relative orientation/distance of the molecular partners. In consequence, often a constant number of intermolecular bonds (usually one or two) with fixed directionality that a single interaction center can provide is assumed in theoretical modeling (Ibenskas and Tornau 2012; Misiunas and Tornau 2012; Ciesielski et al. 2013; Nieckarz and Szabelski 2014; Šimenas et al. 2015; Szabelski et al. 2016, 2017). When, however, the molecular interaction centers (functional groups) are chemically more complex, for example being extended or flexible, the (maximum) number and directionality of intermolecular bonds can change when the molecules are allowed to adopt diverse adsorbed configurations. Specifically, this can occur for those molecules (e.g. tripods) whose continuous in-plane rotation on the adsorbing surface is not strongly limited due to relatively low energy barrier for this type of movement. In consequence, the resulting superstructure can be affected by the size of the interaction centers which can provide effective interaction for a pair of molecules whose relative orientation differs by an angle that is not constant but lies in some interval.

To explore the effect of increased configurational freedom of adsorbed functional building blocks on their surfaceconfined self-assembly in this work we use as an example model tripod molecules equipped with patchy interaction centers with adjustable angular diameter. To simulate these assemblies the off-lattice MC simulation is used in which the adsorbed molecules are allowed to change their positions 
and orientations in a continuous way. The main objective of our study is to asses the effect of lack of corrugation of the molecule-surface potential on the structure formation in the modeled systems. In particular, we focus on the influence of the angular width of the interaction centers on the periodicity and connectivity of the resulting 2D patterns. Moreover, the simulations embrace tripod units differing in symmetry of the backbone, that is $C_{3}$-symmetric molecules and tectons in which molecular arms have unequal lengths or in which neighboring arms meet at angle that is different from 120 degrees. The calculated off-lattice results are compared with their selected counterparts from the lattice MC simulations. The main purpose in this case is to examine whether the off-lattice modeling is able to produce 2D structures having morphologies which are similar to those obtained with the simplified lattice approach. In this context, comparison of the two model representations allows us for the definition of the role of molecule-surface interactions and intermolecular interactions in the 2D pattern formation. Information of this type can be useful in designing molecular systems on solid surfaces, where the substrate and individual properties of the molecular tripod building block can be selected/tuned according to the hints provided in this study.

\section{The model and simulations}

To explore the self-assembly of functional tripods in adsorbed overlayers we used an off-lattice model in which the probe molecules were confined on a plane. These building blocks were modeled as rigid planar structures shown in Fig. 1. Specifically, each molecule was built of the core segment with three attached arms having lengths $a, b$ and $c$. Accordingly, the arms consisted of $a, b$ and $c$ segments and the angles between the arms, $\angle(b, c)$ and $\angle(a, b)$, were equal to $\Psi_{1}$ and $\Psi_{2}$, respectively. All segments, including the core and arm components, had the same diameter, $\sigma$. The terminal arm segments, called further active segments, were equipped with identical patches providing attractive intermolecular interactions. Each associating patch was delimited by a circular sector with angular diameter equal to $\theta$. Intermolecular interactions were allowed only between a pair of active segments.

In the remaining case the interaction was equal to zero and the simple excluded area criterion was used, so that the molecules could not overlap. The interaction between active segments was described by the pair interaction potential, $u_{a s}$ having the following form:

$u_{a s}\left(\mathbf{r}_{i j}, \mathbf{n}_{i}, \mathbf{n}_{j}\right)=u_{s w}\left(r_{i j}\right) \Omega\left(\mathbf{r}_{i j}, \mathbf{n}_{i}, \mathbf{n}_{j}\right)$

where the square-well potential term equals

$u_{s w}\left(r_{i j}\right)=\left\{\begin{array}{cc}\varepsilon & \text { if } \sigma<r_{i j}<\sigma+\Delta \\ 0 & \text { otherwise }\end{array}\right.$

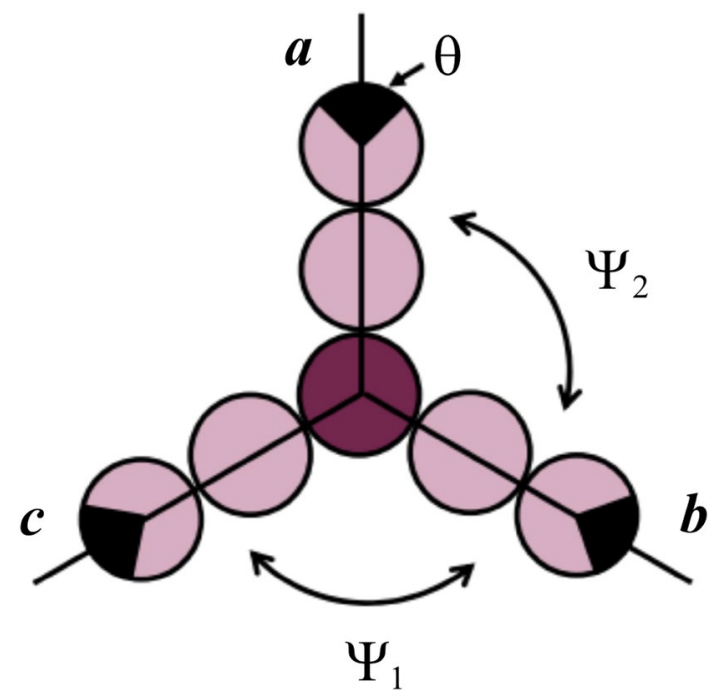

Fig. 1 Schematic structure of the tripod molecule used in the simulations. The molecule consists of the core segment (dark purple) and three arms built of $a, b$ and $c$ segments (light purple), respectively; here $a=b=c=2$. The angles $\Psi_{1}$ and $\Psi_{2}$ determine relative position of the arms $b$ and $c$ and $a$ and $b$, respectively. The interaction centers are delimited by the identical circular sectors (black) with angular diameter equal to $\theta$. (Color figure online)

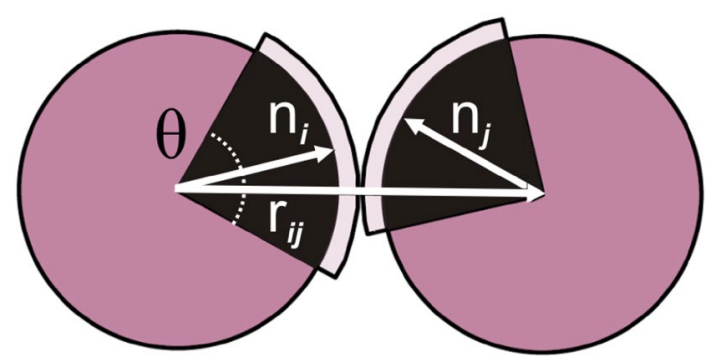

Fig. 2 Schematic representation of a pair of interacting active molecular segments. The vector $\mathbf{r}_{i j}$ points from the center of segment $i$ to the center of segment $j$. The vectors $\mathbf{n}_{i}$ and $\mathbf{n}_{j}$ indicate orientations of the molecules $i$ and $j$. The interaction is possible $(\Omega=1)$ when the vector $\mathbf{r}_{i j}$ connecting the segment centers intersects both circular sectors. The zones colored in light purple are the interaction shells of thickness $\Delta$ and angular diameter $\theta$ (dotted line)

and the orientation-dependent part was defined as

$\Omega\left(\mathbf{r}_{i j}, \mathbf{n}_{i}, \mathbf{n}_{j}\right)=\left\{\begin{array}{l}1 \text { if } \mathbf{r}_{i j} \times \mathbf{n}_{i}>\cos \theta \text { and } \mathbf{r}_{i j} \times \mathbf{n}_{j}>\cos \theta \\ 0 \quad \text { otherwise }\end{array}\right.$

In the above equations the parameters $\Delta$ and $\varepsilon$ are the interaction range and energy, respectively. The meaning of the vectors $\mathbf{r}_{i j}, \mathbf{n}_{i}$ and $\mathbf{n}_{j}$ is explained in Fig. 2 which shows a pair of interacting active segments. According to the assumed conditions (Eq. (3)) the interaction illustrated in Fig. 2. is possible only if the vector connecting the segment centers 
intersects both circular sectors (active zones). If so, when the condition (2) is additionally satisfied, the molecules interact via the square-well potential that is with energy equal to $\varepsilon$.

The simulations were carried out using the conventional MC method in canonical ensemble in which the side of the square simulation box $L$, temperature $T$ and number of molecules $N$ were fixed. In all of the calculations $L$ was set to $96 \sigma$, where $\sigma=1$ was chosen to be the distance unit in our model. To minimize edge effects standard periodic boundary conditions in both planar directions were used. Each MC simulation step (MCS) consisted of an attempt to change position and an orientation of randomly chosen molecule. In this procedure displacements ranging from $0.05 \sigma, 0.10 \sigma$, and $0.15 \sigma$ (non-local moves) were used and random in-plane rotation of a molecule was performed. To make the configuration sampling more effective the conventional Metropolis algorithm was combined with the orientational bias sampling technique (Frenkel and Smit 2001). Moreover, in each MCS an attempt was made to rotate in-plane a randomly selected molecule without translating it (local moves). To equilibrate the modeled systems $10^{6}$ MCS were initially used and subsequent $10^{4}$ MCS served for averaging and calculation of the associated structural characteristics. The energies and temperatures in our models are expressed in units of $\omega$ and $|\omega| / k$ respectively, where $k$ is the Boltzmann constant. All of the simulations were carried out for $\Delta=0.1$ and $\varepsilon=4 \omega$ with $\omega=-1$. The surface coverage, $f$ was defined as $(a+b+c+1) N / \sigma^{2} L^{2}$. Taking into account that $\sigma=1$ the surface coverage can be interpreted as the average number of molecular segments per unit area or per adsorption site in the lattice model described in the following.

The structure formation in adsorbed overlayers simulated with the off-lattice model was analyzed by means of the core-core radial distribution functions (ccrdf) which provide information about the range of ordering in the modeled systems, including local structure (closest coordination) in the vicinity of a single molecule. The ccrdf used here is a measure of the average probability of finding a molecular core at a certain distance away from a given reference molecular core. An additional parameter which we calculated to trace structural changes in the simulated systems was the mean coordination number of a molecule. This quantity was defined as the average number of foreign active segments adsorbed within the interaction zones $(\theta, \Delta)$ of the active segments of a selected molecule.

The simulations were performed for a few representative tripod building blocks differing in size and aspect ratio. Parameters of these diverse test cases are collected in Table 1. The first group of molecules, A was characterized by equal angles between the consecutive arms, that is $\Psi_{1}=\Psi_{2}=120^{\circ}$. The second group, called $\mathbf{B}$ embraced those molecules for which $\Psi_{1}=60^{\circ}$ and $\Psi_{2}=150^{\circ}$.
Table 1 Structural parameters of the tripod building blocks used in the simulations

\begin{tabular}{llll}
\hline Molecule type & $a$ & $b$ & $c$ \\
\hline A: $\Psi_{1}=\Psi_{2}=120^{\circ}$ & 1 & 1 & 1 \\
& 1 & 1 & 2 \\
B: $\Psi_{1}=60^{\circ}, \Psi_{2}=150^{\circ}$ & 1 & 2 & 2 \\
& 2 & 2 & 2 \\
& 1 & 1 & 1 \\
\hline
\end{tabular}

For selected molecules of A-type we also performed the corresponding lattice MC simulations in which triangu-

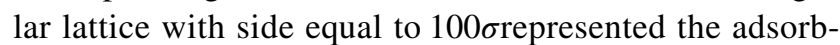
ing (111) crystalline surface. In the simulations of this type, each molecular segment of $\mathbf{A}$ was allowed to occupy one vertex (adsorption site) of the lattice. This condition imposes lattice spacing equal to the distance between a pair of adjacent segments in the molecule of $\mathbf{A}$, equal to $\sigma$. The segments of two adsorbed molecules were not allowed to occupy neighboring lattice sites. The directional interaction between a pair of molecules A was possible only when their active segments occupied next nearest neighbors on the lattice and moreover, the angle between the contributing molecular arms was equal to $120^{\circ}$. In such a case, the interaction energy was equal to $\omega$. The simulations were preformed using the conventional MC simulation scheme in the canonical ensemble, based on the Metropolis sampling (Szabelski et al. 2016). Periodic boundary conditions were imposed in both planar directions. The adsorbed overlayer was equilibrated in a series of attempts of changing positions and orientations of $N$ adsorbed molecules. Each of these attempts constituted one MC step and $5 \times 10^{6} \times N$ MC steps were performed to finalize the simulation. To minimize the risk of trapping the modeled systems in metastable states the cooling procedure was used, in which the temperature, $T$ was gradually decreased from 0.5 to the target value 0.01 using 500 decrements of equal length.

\section{Results and discussion}

To asses the influence of the differently encoded interaction centers we tested a few representative cases in which the angular diameter of the associating patches, $\theta$ was changed. Moreover, we took into account the effect of molecular geometry, that is we studied the self-assembly of molecular tripods having arms of unequal lengths and meeting at angles different from 120 degrees (see Table 1). Let us start the discussion with the simplest tectons of A-type having arms of the same length. Figure 3 presents snapshots of the adsorbed overlayers simulated for the smallest molecule 

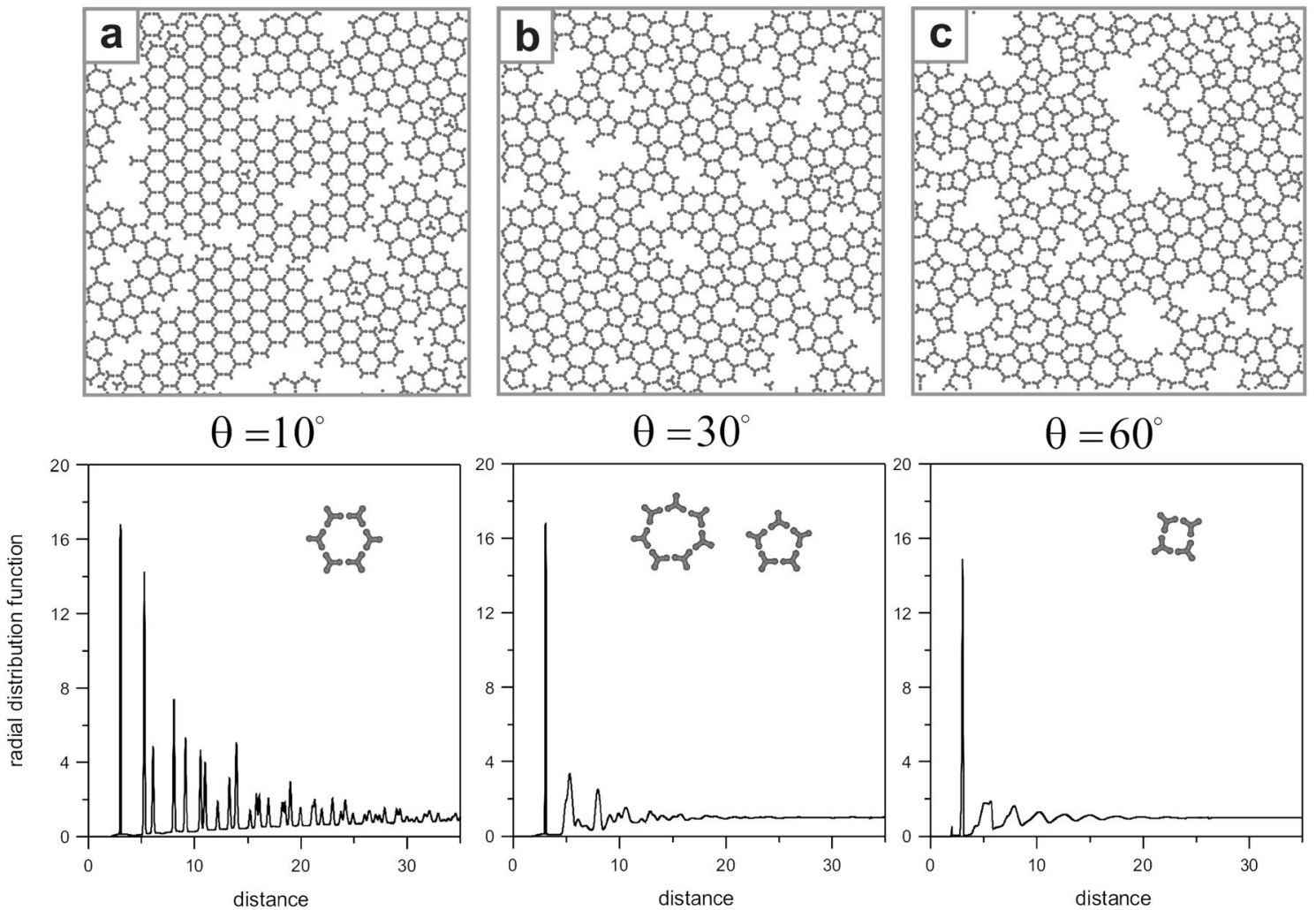

Fig. 3 Snapshots of the adsorbed overlayer comprising 692 molecules of $\mathbf{A}_{111}$ having terminal interaction centers with different angular diameters, $\theta$ (top a-c); $f=0.30, T=0.40, L=96$. The bottom part

$\mathbf{A}_{111}$, for different values of the parameter $\theta$, that is $10^{\circ}, 30^{\circ}$ and $60^{\circ}$. From Fig. 3 it follows that for the molecule $\mathbf{A}_{111}$ with angularly narrow interaction patches $\left(\theta=10^{\circ}\right)$ the selfassembly results in the ordered porous network with regular hexagonal void spaces. The obtained periodic assembly is structurally identical with the network simulated previously using the lattice model MC (Szabelski et al. 2016). In that approach the molecules were adsorbed on a triangular lattice and, contrary to the present case, they could adopt only a few-in plane orientations imposed by the symmetry of the lattice. Moreover, in our previous modeling the interaction between a pair of tripod molecules was possible when their arms were collinear. This condition, as demonstrated in Fig. 3, is equivalent to the presence of terminal interaction patches which are sufficiently narrow to enforce the molecular arms to align parallel and form two-fold nodes. Such type of on-surface ordering was observed experimentally for numerous tripod molecules equipped with terminal carboxylic groups interacting via highly directional hydrogen bonds (Dienstmaier et al. 2010).

An interesting change in the morphology of the adsorbed overlayer can be observed when the angular diameter, $\theta$ is increased. This can be seen in the central snapshot from Fig. $3\left(\theta=30^{\circ}\right)$ in which frequently occurring structural shows the corresponding radial distribution functions and characteristic structural motifs (pores) occurring in the snapshots (a-c); see the insets

motifs are the deformed pentagonal and heptagonal pores shown in the inset below. The increased value of $\theta$ enables the molecular arms of $\mathbf{A}_{111}$ to meet at angles which are slightly different from $180^{\circ}$ - characterizing the regular hexagonal pores (see the inset, $\theta=10^{\circ}$ ). In consequence, the obtained network is aperiodic and it is a mixture of connected pores whose rims are built of five, six and seven molecules. This pore distribution changes markedly when the angular diameter is further increased, up to $60^{\circ}$ (right panel). Specifically, in the corresponding adsorbed system a new pore type, formed by four molecules, can be identified (see the inset). The additional four-membered pores increase considerably structural heterogeneity of the obtained network. Here, (often deformed) pores of four types create the disordered network which lacks long-range order. The structures simulated for $\theta=30^{\circ}$ and $\theta=60^{\circ}$ are very similar to the experimental results on surface-confined polymerization of halogenated polyaromatic molecules. For example, the structural motifs which have been observed for 1,3,5-tris(4-bromophenyl)benzene (TBB) on $\mathrm{Cu}(111)$ (Gutzler et al. 2009) are identical with those predicted in this study (Fig. 3c). A common feature of the molecular networks shown in Fig. 3 is that the contributing molecules always form two-fold nodes, that is intermolecular 
interactions always involve a pair of molecular arms. Nodes in which three or more arms meet are absent or very rare. In consequence, a single molecule has usually three neighboring partners in its direct vicinity, regardless of $\theta$. This can be clearly seen in the ccrdf plotted in the bottom part of Fig. 3. For all of these systems the largest peak occurs at the distance equal to about three. This value characterizes the core-core distance between a pair of molecules whose contacting arms are in (nearly) collinear alignment. Further analysis of the core-core rdfs shows the decreasing range of ordering that is induced by the increase in $\theta$. For the highly ordered network simulated for $\theta=10^{\circ}$ the corresponding rdf comprises several narrow peaks which appear even at the distances exceeding 20 . These peaks are the contributions from the increasing regular shells of molecules surrounding a selected molecule. In the case of the systems with $\theta=30^{\circ}$ and $\theta=60^{\circ}$ the calculated rdfs are much less peaked and reach a constant value yet at $\sim 15$. This property confirms the lack of long-range order which can be seen in the central and right snapshots from Fig. 3 .

Figure 4 presents the effect of temperature on the mean coordination number of a molecule of $\mathbf{A}_{111}$ equipped with the associating patches having different angular diameter (692 molecules, $f=0.3$ ). As it follows from the figure, the coordination number of $\mathbf{A}_{111}$ increases with decreasing temperature and at $T=0.4$ it reaches about $2.48\left(\theta=10^{\circ}\right)$, $2.63\left(\theta=30^{\circ}\right)$ and $2.79\left(\theta=60^{\circ}\right)$. At this temperature most of the molecules form three connections with their neighbors, as seen in Fig. 3a-c, so that the above values are close

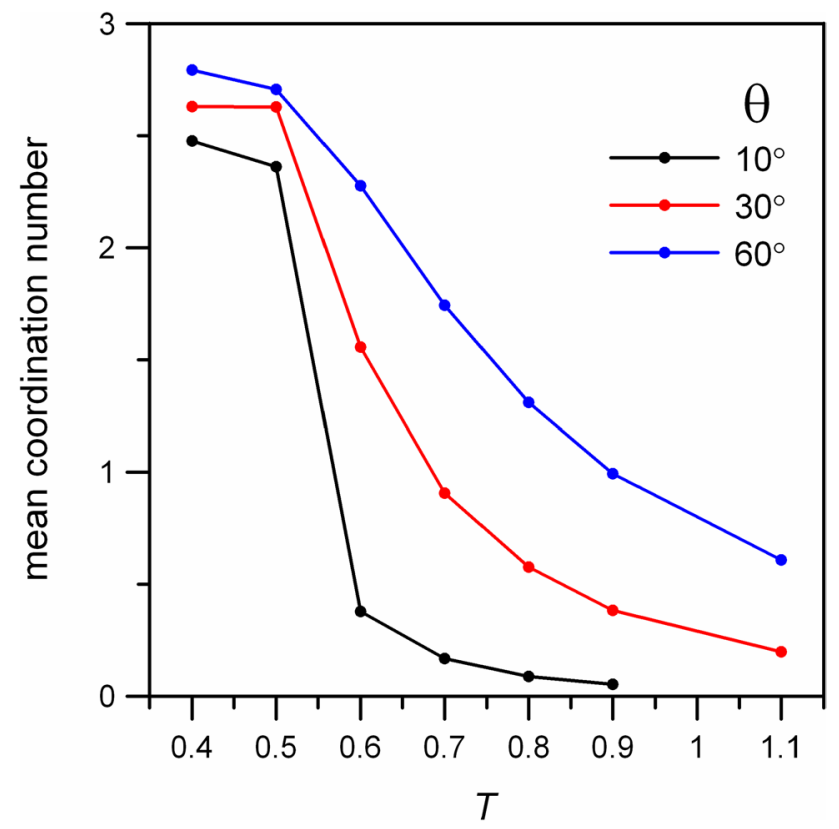

Fig. 4 Effect of temperature on the mean coordination number of the tripod building block $\mathbf{A}_{111}$ with the interaction patches having different angular diameter, $\theta(N=692, f=0.3)$ but not equal to three. The incomplete coordination of $\mathbf{A}_{111}$ originates mainly from the finite-size domains which comprise numerous peripheral molecules. From Fig. 4 it can be also seen that the coordination number at $T=0.4$ increases slightly when the associating patches become angularly wider. This effect results from the increased ability of the molecules with larger $\theta$ to create three-fold nodes, a few of which can be identified in Fig. 3c. An interesting observation from Fig. 4 is that the network heterogeneity that is induced by the increasing angular diameter (see the snapshots from Fig. 3) is a factor which reduces substantially sharpness of the structural transition occurring in the modeled systems. This can be clearly seen when comparing the curves from Fig. 4, of which only the one calculated for $\theta=10^{\circ}$ exhibits a steep increase (at $T=0.6$ ). In the two remaining cases $\left(\theta=30^{\circ}\right.$ and $\left.\theta=60^{\circ}\right)$, the formation of the networks proceeds much less rapidly when the adsorbed overlayer is cooled down. Here, due to the less restricted angular requirement for the bond formation, the molecules are able to create porous structures yet at higher temperatures and these structures are more "flexible" in terms of incorporating new molecular bricks.

Further increase in the angular diameter, $\theta$ results in the emergence of new adsorbed structures formed by $\mathbf{A}_{111}$. This can be seen in Fig. 5 which shows the snapshots simulated for $\theta$ equal to $75^{\circ}, 120^{\circ}$ and $150^{\circ}$. For the first value we can observe the creation of ordered porous networks (panel a) with hexagonal void spaces. An important feature which makes this superstructure different from that one obtained for $\theta=10^{\circ}$ (c.f. Fig. 3a) is the connectivity and pore architecture. Specifically, the network simulated for $\theta=75^{\circ}$ is sustained by the three-fold nodes which are built by three composite molecules. Accordingly, the pore rims are formed by three molecules of $\mathbf{A}_{111}$ (see the inset) and the network is characterized by a rhombic unit cell with side equal to about 2.77. The regular geometry of the aforementioned pores becomes disturbed when the angular diameter increases further and this effect is shown in Fig. $5 \mathrm{~b}$ for $\theta=120^{\circ}$. The network presented in this panel comprises three-membered pore-type structural units, one of which is shown in the inset as an example. The disturbed geometry of these pores originates from the slightly shifted positions of the molecular cores which do not occupy vertices of a equilateral triangle. Moreover, the three molecules forming such an irregular pore can have different in-plane orientations, so that their corresponding arms are not collinear, as it was often observed for $\theta=75^{\circ}$. A noticeable change in the structure of the adsorbed overlayer occurs for $\theta=150^{\circ}$ (Fig. 5c). In this case, the porosity of the molecular assembly is completely lost and, instead, irregular compact clusters are formed. To quantity the effect of the angular diameter on the self-assembly of $\mathbf{A}_{111}$ we calculated the corresponding mean coordination numbers. These dependencies, determined for the two 
Fig. 5 Snapshots of the adsorbed overlayer comprising 692 molecules of $\mathbf{A}_{111}$ simulated for the different values of the angular diameter, $\theta ; f=0.30, T=0.80, L=96$; $(\mathbf{a}-\mathbf{c})$. The inset in part a shows a magnified fragment of the network with the unit cell marked in red. The small insets in a and b present basic structural units (pores) of the networks. Effect of the angular diameter on the mean coordination number calculated at two temperatures (d). The color codes used in $\mathbf{d}$ correspond to the exemplary configurations shown in $(\mathbf{a}-\mathbf{c})$. (Color figure online)
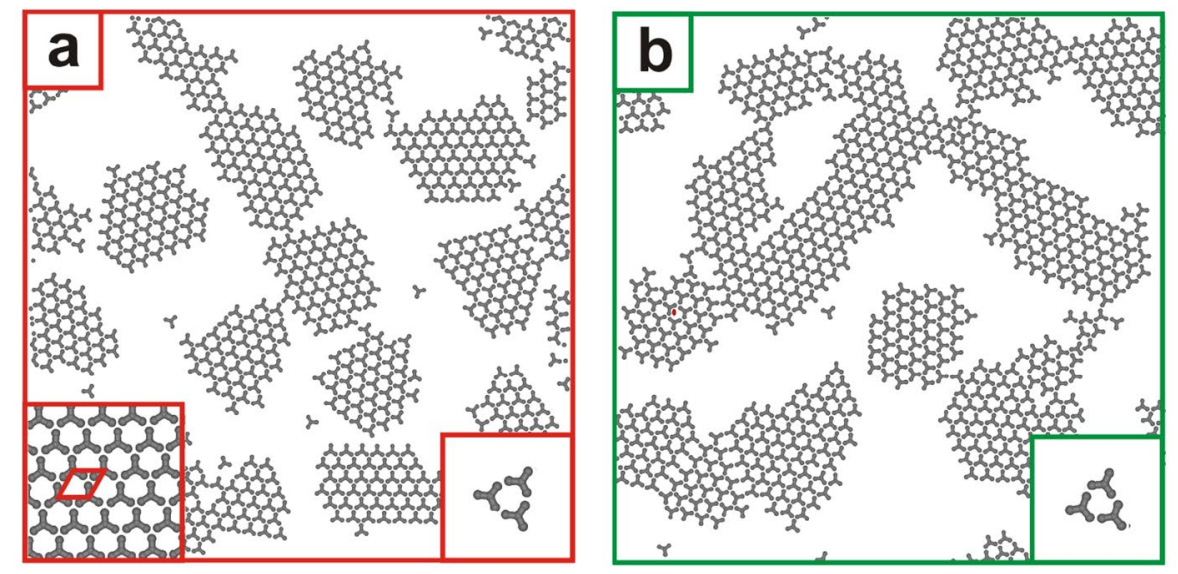

$$
\theta=75^{\circ}
$$

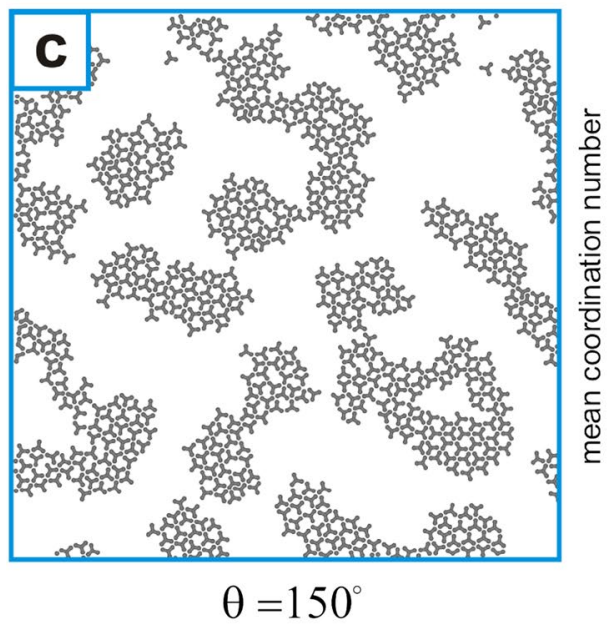

$$
\theta=120^{\circ}
$$

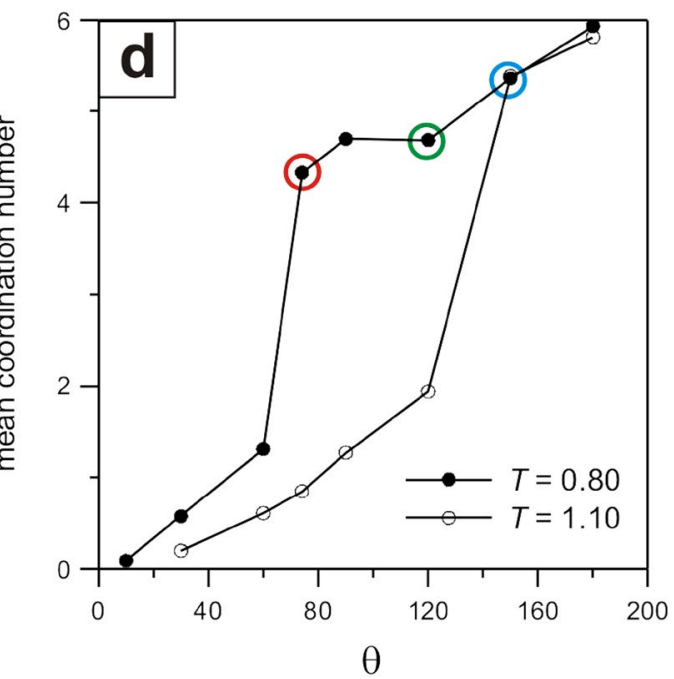

temperatures, $T=0.80$ and 1.10, are shown in Fig. $5 \mathrm{~d}$. As we can observe there, for the lower temperature, the mean coordination number increases rapidly when $\theta$ exceeds $60^{\circ}$ and it stays at about five until $\theta=120^{\circ}$. The molecules of $\mathbf{A}_{111}$ self-assembly then into finite-sized domains of the ordered network-type phases shown in panels ( $a$ and $b$ ). In these networks (infinite and defect free) a molecule of $\mathbf{A}_{111}$ is coordinated by six partners. The lowered coordination number, equal to about five, results from the formation of the dispersed domains which comprise numerous peripheral (partially coordinated) molecules. At the higher temperature, $T=1.10$, the formation of the networks is largely suppressed, so that the coordination number calculated for $75^{\circ} \leq \theta \leq 120^{\circ}$ is considerably smaller, as compared to $T=0.80$. Further increase in the angular diameter triggers the formation of the disordered compact clusters (panel c) which are characterized by the average coordination number which approaches six at $\theta=180^{\circ}$.

To explore the possibility of directing the self-assembly of the molecules with reduced symmetry $\left(\mathbf{A}_{112}\right.$ and $\left.\mathbf{A}_{122}\right)$ into the networks stabilized by the newly observed three-fold nodes (c.f. Fig. 5a, b) we performed additional simulations, results of which are shown in Fig. 6. The calculations we run for $\theta=75^{\circ}$ demonstrated the formation of porous phases comprising hexagonal void spaces with different aspect ratios. The networks presented in panels ( $a$ and $b$ ) were characterized by the exemplary parallelogram $3.63 \times 2.76$ and $4.54 \times 3.58$ unit cells (red lines), respectively which corresponds to the density equal to 0.53 and 0.45 . For comparison, in panel (d) we showed also the network formed by the $C_{3}$-symmetrical molecule of $\mathbf{A}_{222}$ which is the isotropically enlarged version of the unit $\mathbf{A}_{111}$. For this larger building block, like for $\mathbf{A}_{111}$, the unit cell had a rhombic shape but with longer side equal to about 4.46. The corresponding network density was equal to 0.40 . The long-range order observed in the porous phases from Fig. 6. vanishes when the angular diameter is increased. This effect produces disordered clusters which, however, are more frequently interconnected, as compared to the isolated domains formed by $\mathbf{A}_{111}$ (see Fig. 5c).

In the next example we present the results calculated for the molecules of type $\mathbf{B}$ having unequal arm-arm angles, 

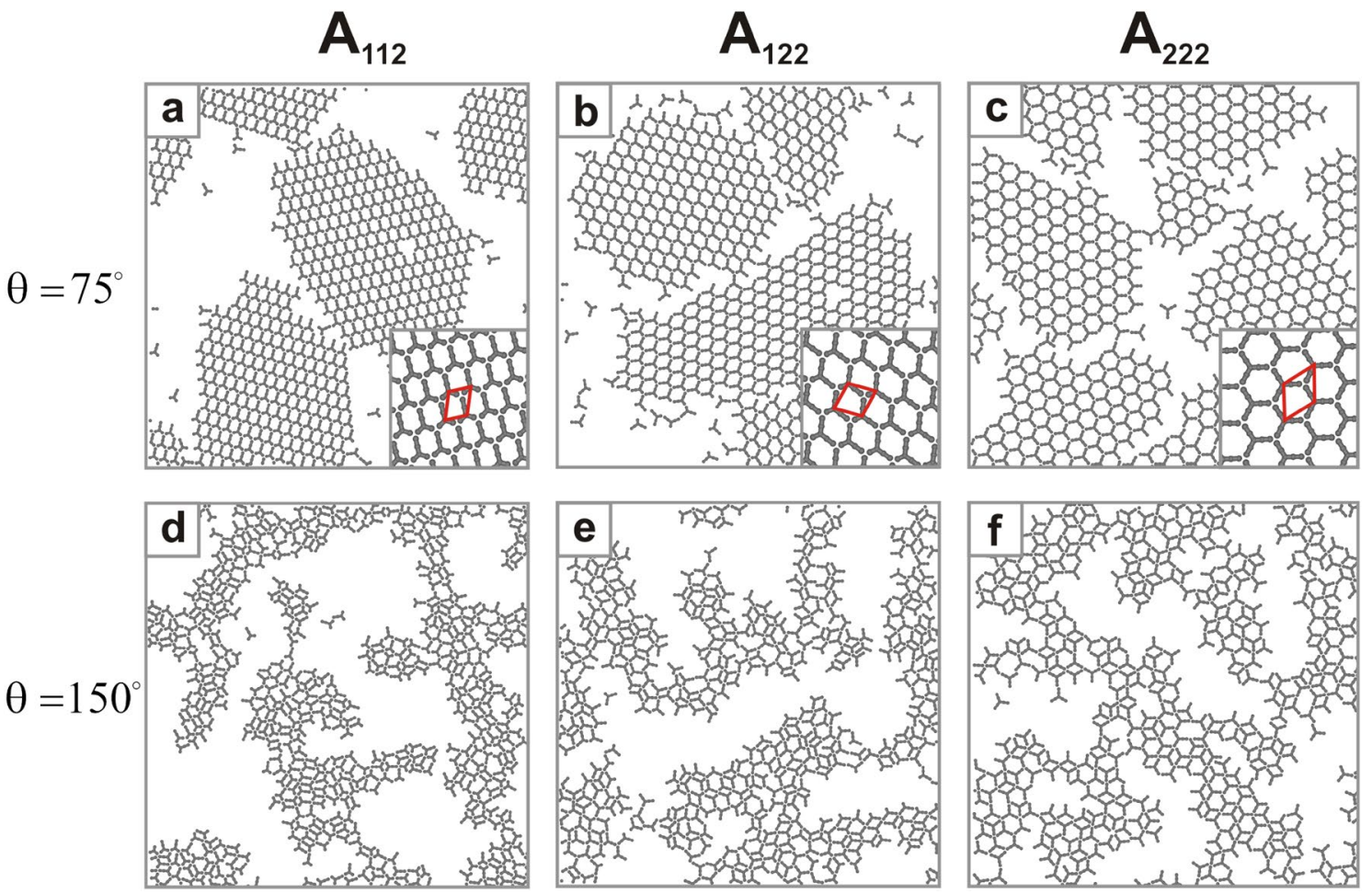

Fig. 6 Snapshots of the adsorbed overlayer comprising 553 (a, d), 461 (b, e) and 395 tripod-shaped building blocks A differing in size and aspect ratio simulated at $T=0.8$ for two values of the angular diameter, $\theta$. The insets in the top part are magnified fragments of the corresponding networks. Parallelogram (a, b) and rhombic (c) unit cells of these superstructures are delimited by the red lines. (Color figure online)

at vertices of the pores. The modified angular position of the arms in $\mathbf{B}$ makes these molecules behave like ditopic rods bearing sufficiently wide $(\theta)$ terminal interaction centers. This comparison is the most adequate for the elongated molecule $\mathbf{B}_{112}$ (see Fig. 7c) which creates networks with distinct square pores. The ordered porous networks of $\mathbf{B}$ formed at $\theta=75^{\circ}$ were no longer observed when the angular diameter was increased up to $150^{\circ}$. For this angular diameter, more $\left(\mathbf{B}_{111}\right)$ or less $\left(\mathbf{B}_{112}\right)$ compact disordered aggregates were formed, highlighting the similar effect of $\theta$ on the structure formation, as compared to the unit A (c.f. Fig. 6).

The porous networks of A sustained by the three-fold nodes (Fig. 6) exhibit high symmetry and they resemble molecular superstructures which have been formed on structured substrates playing the role of a guide for the self-assembly (Shang et al. 2015; Ciesielski et al. 2013). This refers especially to the (111) metallic surfaces and graphite-substrates often imposing a few in-plane orientations of adsorbed molecules and occupation of discrete positions on the surface. As we demonstrated previously, such adsorbed systems can be successfully modeled using the coarse-grained MC lattice models (Ciesielski et al. 2013; Nieckarz and Szabelski 2013; Szabelski et al. 2016). We use this approach here to examine whether the networks created 

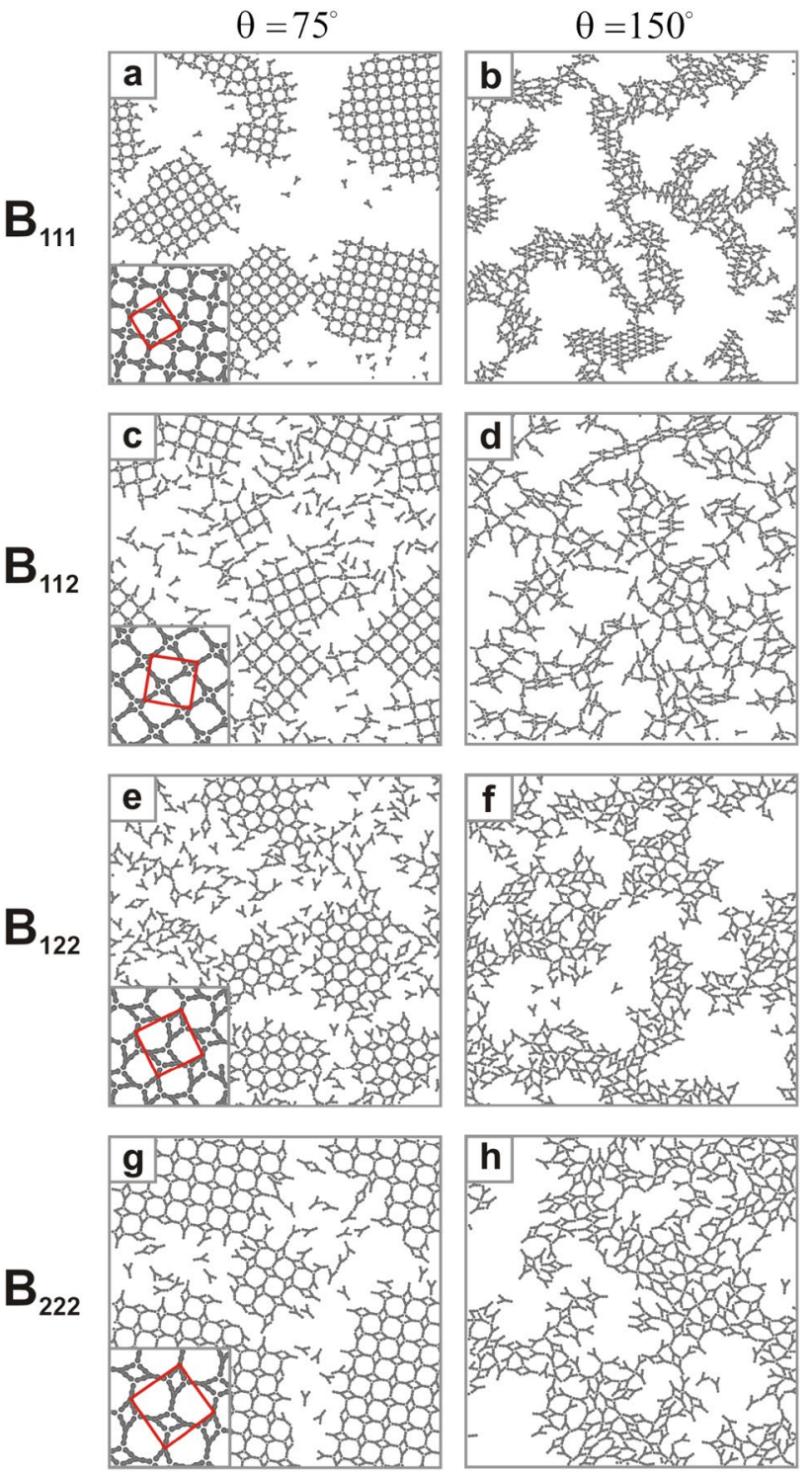

Fig. 7 Snapshots of the adsorbed overlayers comprising 691 (a, b), 553 (c, d), $461(\mathbf{e}, \mathbf{f})$ and $395(\mathbf{g}, \mathbf{h})$ molecules of type B ( $f=0.3, T=0.8$ ) obtained for two values of the angular diameter, $\theta$. The red lines delimit the square unit cells of the corresponding networks. (Color figure online)

by $\mathbf{A}$ with $\theta=75^{\circ} \mathrm{can}$ be also obtained using the less computationally costly lattice $\mathrm{MC}$ method for the triangular grid of adsorption sites. To that purpose we performed the lattice simulations for the adsorbed overlayers $(L=100)$ comprising 625, 500, 416 and 357 molecules of $\mathbf{A}_{111}, \mathbf{A}_{112}, \mathbf{A}_{122}$ and $\mathbf{A}_{222}$, respectively, maintaining constant surface coverage $f=0.25$. This common value of $f$ was carefully chosen to ensure unrestricted growth of the networks formed by the largest unit $\mathbf{A}_{222}$. Figure 8 presents snapshots simulated for these four tectons at $T=0.01$.

As it can be seen in Fig. 8, the simulated networks are isostructural with their counterparts obtained by means of the off-lattice MC simulations. For all of the considered molecular geometries, the corresponding network architectures agree with those shown in Fig. 6a-c. This refers to the internal structure of the three-fold nodes (see the inset in panel a) and to the periodicity of the obtained porous superstructures. One effect which can be seen in Fig. 8 is that the underlying lattice facilitates largely the self-assembly of extended domains formed by $\mathbf{A}$. The limited number of in-plane orientations of an adsorbed molecule (two for $\mathbf{A}_{111}$ and $\mathbf{A}_{222}$ and six for $\mathbf{A}_{112}$ and $\mathbf{A}_{122}$ ) results in the fast growth of the networks. This process involves mostly incorporation of new molecules of $\mathbf{A}$ at the periphery of a growing domain. For the lowered configurational freedom of $\mathbf{A}$ imposed in the lattice model, the aforementioned incorporation occurs much easier and it is additionally guided by the underlying lattice. As a consequence, contrary to the off-lattice results, the domains formed by $\mathbf{A}$ can have only a few discrete orientations with respect to the substrate (differing by a multiple of 60 degrees). This can be seen in Fig. 8. The networks simulated using the lattice model are characterized by the unit cells with the following parameters: $\mathbf{A}_{111}$ and $\mathbf{A}_{222}$-rhombic with side equal to $2 \sqrt{3}$ and $3 \sqrt{3}$, respectively; $\mathbf{A}_{112}$ - parallelogram $\left(\sqrt{19} \times 2 \sqrt{3}, 67^{\circ}\right)$ and $\mathbf{A}_{122}$ - parallelogram $\left(\sqrt{19} \times 3 \sqrt{3}, 53^{\circ}\right)$. The associated network densities are equal to: $0.385,0.361,0.330$ and 0.300 and they are lower than the corresponding values obtained with the off-lattice model. The difference between the results obtained using the two types of models (lattice and off-lattice) originate from the differently limited minimal distances separating a pair of molecular segments. For the continuous model this distance is equal to $\sigma=1$ while in the lattice model the closest configuration of two molecules is that their segments occupy next nearest neighbor positions on the triangular lattice, separated by $\sqrt{3}$ (see the inset in Fig. 8a). The increased value of the minimal intersegment distance assumed in the lattice model (by factor of about 1.73) results in the larger unit cells and thus lower densities of the simulated networks, as compared to the offlattice model. Nonetheless, the architectures of the porous phases obtained using the two methods are identical. This result highlights usefulness of the lattice MC modeling in predicting new molecular patterns sustained by short-range interactions. In particular, our calculations demonstrate that in certain cases 2D molecular porous superstructures can be reproduced accurately using the less computationally costly lattice MC simulations.

\section{Conclusions}

The results of our theoretical investigations demonstrated that the surface-assisted self-assembly of tripod-shaped functional building blocks can be largely affected by the 
Fig. 8 Snapshots of the adsorbed overlayer comprising 625 (a), 500 (b), 416 (c) and 357 (d) molecules of type $\mathbf{A}$ obtained with the MC simulations on a $100 \times 100$ triangular lattice $(f=0.25, T=0.01)$. The inset in (a) shows the nextnearest interactions (red arrows) between molecules forming the three-fold node. The insets to (b-d) are the magnified fragments of the corresponding networks with the unit cells marked by the red lines. (Color figure online)
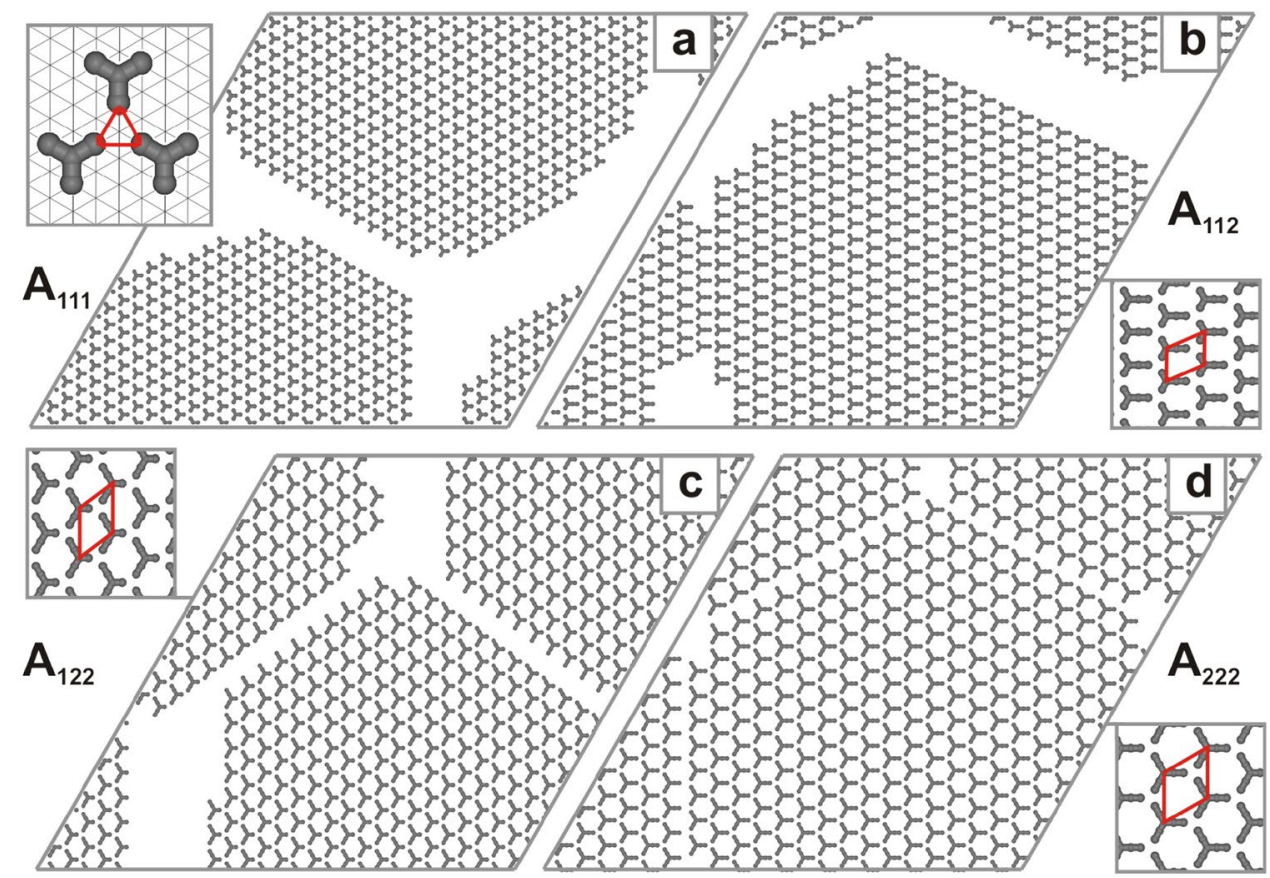

intrinsic properties of the peripheral molecular interaction centers. The MC simulations performed for the selected systems showed that the angular diameter of the associating patches, $\theta$ plays a decisive role in the structure formation and it is responsible for the emergence of network-type ordered phases. For example, extended networks with regular hexagonal void spaces were obtained for the molecule $\mathbf{A}_{111}$ when it was equipped with angularly narrow patches $\left(\theta=10^{\circ}\right)$. The results calculated for this tecton proved that the narrow interaction patches enhance the self-assembly of porous networks which are isostructural with their counterparts observed in the corresponding lattice MC simulations (Szabelski et al. 2016). New types of porous networks were obtained for the increased value of the angular diameter $\left(\theta=75^{\circ}\right)$. In this case, for the molecules of A-type, the self-assembly resulted in the porous networks sustained by the three-fold nodes. Our separate calculations showed that porous networks with identical type of connectivity (three-fold nodes) and periodicity can be obtained with the less computationally demanding lattice MC simulation method (Fig. 8). On the other hand, the off-lattice simulations carried out for the molecules of B-type having lowered symmetry predicted the formation of new type of network with square-grid architecture and rounded square pores. As we observed, further increasing of the angular diameter $\left(\theta=150^{\circ}\right)$ resulted in the disordered assemblies, regardless of the tecton type.

The MC results discussed in this study can be helpful in designing new molecular systems on solid surfaces comprising tripod-shaped functional organic molecules. This refers to the adsorbed overlayers sustained by intermolecular interactions such as, for example, hydrogen and halogen bonding. Our theoretical predictions enable linking the individual properties of the tritopic building blocks with the morphology of the corresponding superstructures formed under 2D confinement. This information can facilitate tuning of molecular properties (size, aspect ratio, functional groups) aiming at the self-assembly of adsorbed networks with predefined structural properties and physico-chemical functions.

Acknowledgements This work was supported by the National Science Centre, Poland Research Grant 2015/17/B/ST4/03616.

Open Access This article is distributed under the terms of the Creative Commons Attribution 4.0 International License (http://creativeco mmons.org/licenses/by/4.0/), which permits unrestricted use, distribution, and reproduction in any medium, provided you give appropriate credit to the original author(s) and the source, provide a link to the Creative Commons license, and indicate if changes were made.

\section{References}

Adisoejoso, J., Tahara, K., Lei, S., Szabelski, P., Rżysko, W., Inukai, K., Blunt, M.O., Tobe, Y., De Feyter, S.: One building block, two different nanoporous self-assembled monolayers: a combined STM and Monte Carlo study. ACS Nano 6, 897-903 (2012)

Akimenko, S.S., Gorbunov, V.A., Myshlyavtsev, A.V., Fefelov, V.F.: Self-organization of monodentate organic molecules on a solid surface-a Monte Carlo and transfer-matrix study. Surf. Sci. 639, 89-95 (2015)

Bertrand, H., Silly, F., Teulade-Fichou, M.P., Tortech, L., Fichou, D.: Locking the free-rotation of a prochiral star-shaped guest molecule inside a two-dimensional nanoporous network by introduction of chlorine atoms. Chem. Commun. 47, 10091-10093 (2011) 
Bischoff, F., He, Y., Seufert, K., Stassen, D., Bonifazi, D., Barth, J.V., Auwärter, W.: Tailoring large pores of porphyrin networks on $\mathrm{Ag}(111)$ by metal-organic coordination. Chem. Eur. J. 22, 1529815306 (2016)

Centres, P.M., Ramirez-Pastor, A.J.: Configurational entropy of adsorbed rigid rods: theory and Monte Carlo simulations. Phys. A 388, 2001-2019 (2009)

Ciesielski, A., Szabelski, P.J., Rżysko, W., Cadeddu, A., Cook, T.R., Stang, P.J., Samorì, P.: Concentration-dependent supramolecular engineering of hydrogen-bonded nanostructures at surfaces: predicting self-assembly in 2D. J. Am. Chem. Soc. 135, 6942-6950 (2013)

Copie, G., Cleri, F., Makoudi, Y., Krzeminski, C., Berthe, M., Palmino, F., Grandidier, B.: Surface-induced optimal packing of twodimensional molecular networks. Phys. Rev. Lett. 114, 6610166106 (2015)

De Feyter, S., De Schryver, F.C.: Self-assembly at the liquid/solid interface: STM reveals. J. Phys. Chem. B 109, 4290-4302 (2005)

Dienstmaier, J.F., Mahata, K., Walch, H., Heckl, W.M., Schmittel, M., Lackinger, M.: On the scalability of supramolecular networkshigh packing density vs optimized hydrogen bonds in tricarboxylic acid monolayers. Langmuir 26, 10708-10716 (2010)

Fan, Q., Wang, C., Han, Y., Zhu, J., Kuttner, J., Hilt, G., Gottfried, J.M.: Surface-assisted formation, assembly, and dynamics of planar organometallic macrocycles and zigzag shaped polymer chains with C-Cu-C bonds. ACS Nano 8, 709-718 (2014)

Fan, Q., Dai, J., Wang, T., Kuttner, J., Hilt, G., Gottfried, J.M., Zhu, J.: Confined synthesis of organometallic chains and macrocycles by $\mathrm{Cu}-\mathrm{O}$ surface templating. ACS Nano 10, 3747-3754 (2016)

Frenkel, D., Smit, B.: Understanding molecular simulation. Academic Press, Cambridge (2001)

Gorbunov, V.A., Akimenko, S.S., Myshlyavtsev, A.V.: Cross-impact of surface and interaction anisotropy in the self-assembly of organic adsorption monolayers: a Monte Carlo and transfer-matrix study. Phys. Chem. Chem. Phys. 19, 17111-17120 (2017)

Gutzler, R., Walch, H., Eder, G., Kloft, S., Heckl, W.M., Lackinger, M.: Surface mediated synthesis of 2D covalent organic frameworks: 1,3,5-tris(4-bromophenyl)benzene on graphite (001), $\mathrm{Cu}(111)$, and $\mathrm{Ag}(110)$. Chem. Commun. 29, 4456-4458 (2009)

Ibenskas, A., Tornau, E.E.: Statistical model for self-assembly of trimesic acid molecules into homologous series of flower phases. Phys. Rev. E 86, 51118-51130 (2012)

Kaposi, T., Joshi, S., Hoh, T., Wiengarten, A., Seufert, K., Paszkiewicz, M., Klappenberger, F., Ecija, D., Dordević, L., Marangoni, T., Bonifazi, D., Barth, J.V., Auwärter, W.: Supramolecular spangling, crocheting, and knitting of functionalized pyrene molecules on a silver surface. ACS Nano 10, 7665-7674 (2016)

Kasperski, A., Nieckarz, D., Szabelski, P.: Structure formation in adsorbed overlayers comprising functional cross-shaped molecules: a Monte Carlo study. Surf. Sci. 641, 269-277 (2015)

Kudernac, T., Lei, S., Elemans, J.A.A.W., De Feyter, S.: Two-dimensional supramolecular self-assembly: nanoporous networks on surfaces. Chem. Soc. Rev. 38, 402-421 (2009)

Kühne, D., Klappenberger, F., Decker, R., Schlickum, U., Brune, H., Klyatskaya, S., Ruben, M., Barth, J.V.: Self-assembly of nanoporous chiral networks with varying symmetry from sexiphenyldicarbonitrile on $\mathrm{Ag}(111)$. J. Phys. Chem. C 113, 17851-17859 (2009a)

Kühne, D., Klappenberger, F., Decker, R., Schlickum, U., Brune, H., Klyatskaya, S., Ruben, M., Barth, J.V.: High-quality 2D metalorganic coordination network providing giant cavities within mesoscale domains. J. Am. Chem. Soc. 131, 3881-3883 (2009b)

Li, Y., Lin, N.: Combined scanning tunneling microscopy and kinetic Monte Carlo study on kinetics of $\mathrm{Cu}$-coordinated pyridyl-porphyrin supramolecular self-assembly on a $\mathrm{Au}(111)$ surface. Phys. Rev. B 84, 125418 (2011)

Li, N., Zhang, X., Gu, G.C., Wang, H., Nieckarz, D., Szabelski, P., He, Y., Wang, Y., Lü, J.T., Tang, H., Peng, L.M., Hou, S.M., Wu, K., Wang, Y.F.: Sierpiński-triangle fractal crystals with the $\mathrm{C}_{3 \mathrm{v}}$ point group. Chin. Chem. Lett. 26, 1198-1202 (2015)

Liu, X.H., Wang, D., Wan, L.J.: Surface tectonics of nanoporous networks of melamine-capped molecular building blocks formed through interface schiff-base reactions. Chem. Asian. J. 8, 24662470 (2013)

Matoz-Fernandez, D.A., Linares, D.H., Ramirez-Pastor, A.J.: Critical behavior of long straight rigid rods on two-dimensional lattices: theory and Monte Carlo simulations. J. Chem. Phys. 128, 214902 (2008)

Misiunas, T., Tornau, E.E.: Ordered assemblies of triangular-shaped molecules with strongly interacting vertices: phase diagrams for honeycomb and zigzag structures on triangular lattice. J. Phys. Chem. B 116, 2472-2482 (2012)

Nieckarz, D., Szabelski, P.: Understanding pattern formation in 2D metal-organic coordination systems on solid surfaces. J. Phys. Chem. C 117, 11229-11241 (2013)

Nieckarz, D., Szabelski, P.: Simulation of the self-assembly of simple molecular bricks into Sierpiński triangles. Chem. Commun. 50, 6843-6845 (2014)

Shang, J., Wang, Y., Chen, M., Dai, J., Zhou, X., Kuttner, J., Hilt, G., Shao, X., Gottfried, J.M., Wu, K.: Assembling molecular Sierpiński triangle fractals. Nat. Chem. 7, 389-393 (2015)

Shi, Z., Liu, J., Lin, T., Xia, F., Liu, P.N., Lin, N.: Thermodynamics and selectivity of two-dimensional metallo-supramolecular selfassembly resolved at molecular scale. J. Am. Chem. Soc. 133, 6150-6153 (2011)

Šimenas, M., Ibenskas, A., Tornau, E.E.: Coronene molecules in hexagonal pores of tricarboxylic acids: a Monte Carlo study. J. Phys. Chem. C 19, 20524-20534 (2015)

Sun, Q., Cai, L., Ma, H., Yuan, C., Xu, W.: On-surface construction of a metal-organic Sierpiński triangle. Chem. Commun. 51, 14164-14166 (2015)

Szabelski, P., De Feyter, S., Drach, M., Lei, S.: Computer simulation of chiral nanoporous networks on solid surfaces. Langmuir 26, 9506-9515 (2010)

Szabelski, P., Rżysko, W., Nieckarz, D.: Directing the self-assembly of tripod molecules on solid surfaces: a Monte Carlo simulation approach. J. Phys. Chem. C 120, 13139-13147 (2016)

Szabelski, P., Nieckarz, D., Rżysko, W.: Influence of molecular shape and interaction anisotropy on the self-assembly of tripod building blocks on solid surfaces. Colloids Surf. A 532, 522-529 (2017)

Tahara, K., Furukawa, S., Ujii, H., Uchino, T., Ichikawa, T., Zhang, J., Mamdouh, W., Sonoda, M., De Schryver, F.C., De Feyter, S., Tobe, Y.: Two-dimensional porous molecular networks of dehydrobenzo[12] annulene derivatives via alkyl chain interdigitation. J. Am. Chem. Soc. 128, 16613-16625 (2006)

Tahara, K., Yamaga, H., Ghijsens, E., Inukai, K., Adisoejoso, J., Blunt, M.O., De Feyter, S., Tobe, Y.: Control and induction of surfaceconfined homochiral porous molecular networks. Nat. Chem. 3, 714-719 (2011)

Trawny, D., Schlexer, P., Steenbergen, K., Rabe, J.P., Paulus, B., Reissig, H.U.: Dense or porous packing? Two-dimensional self-assembly of star-shaped mono-, bi-, and terpyridine derivatives. Chem. Phys. Chem. 16, 949-953 (2015)

Zhang, X., Li, N., Gu, G.C., Wang, H., Nieckarz, D., Szabelski, P., He, Y., Wang, Y., Xie, C., Shen, Z.Y., Lü, J.T., Tang, H., Peng, L.M., Hou, S.M., Wu, K., Wang, Y.F.: Controlling molecular growth between fractals and crystals on surfaces. ACS Nano $\mathbf{9}$, 11909-11915 (2015) 